\title{
Diagnostic and prognostic value of serum interleukin-16 in patients with gastric cancer
}

\author{
HONGYUN YANG ${ }^{*}$, YANYAN HAN", LELE WU and CHAOJUN WU \\ Department of Clinical Laboratory Medicine, Affiliated Hospital of Taishan \\ Medical University, Tai'an, Shandong 271000, P.R. China
}

Received January 1, 2017; Accepted August 8, 2017

DOI: $10.3892 / \mathrm{mmr} .2017 .7688$

\begin{abstract}
Gastric cancer (GC) is one of the major leading causes of cancer-associated mortality worldwide. Serum biomarkers have a vital role in diagnosis and prognosis of GC, and interleukin (IL)-16 may serve as a useful biomarker with prognostic value for human cancers. The current study aimed to evaluate the expression level of serum IL-16 in patients with GC, and evaluate the diagnostic and prognostic value of IL-16. ELISA was performed determine the serum IL-16 levels in patients with GC and healthy controls. Receiver operator curve analysis was performed to evaluate the diagnostic and prognostic potential value of serum IL-16 in GC diagnosis. Migration and invasion assays were performed using cells with IL-16 small interfering RNA (siRNA) knockdown. The results demonstrated that serum IL-16 levels were significantly higher in GC samples than in healthy controls, and increased serum IL-16 levels were significantly associated with tumor recurrence and poor prognosis. Knockdown of IL-16 significantly suppressed the migration and invasion of GC cells. In conclusion, the current results indicate that serum IL-16 levels may have diagnostic and prognostic value for patient with GC.
\end{abstract}

\section{Introduction}

Gastric cancer remains the second leading cause of cancer-associated mortality worldwide and is the fourth most common malignancy. East Asian countries account for $\sim 50 \%$ of the incidence of gastric cancer (GC), and $\sim 1$ million new cases and 0.7 million deaths per year worldwide (1). Due to the limited of techniques for GC screening, the majority of

Correspondence to: Dr Chaojun Wu, Department of Clinical Laboratory Medicine, Affiliated Hospital of Taishan Medical University, 706 Tai'an Street, Tai'an, Shandong 271000, P.R. China E-mail: chaojunwu1968@126.com

\section{${ }^{*}$ Contributed equally}

Key words: gastric cancer, biomarker, interleukin-16, diagnosis, prognosis patients with GC are diagnosed at an advanced stage, and the prognosis for patients with advanced $\mathrm{GC}$ remains poor.

Patients with GC have considerably lower survival rates than those with other gastroenterological tract cancers, except for cancer of the esophagus, and the 5-year survival rate of patients with localized disease (stages I, II, N0; 63.2\%) decreases to $28.4 \%$ as the cancer spreads to regional lymph nodes (stages II, III, N1-N3), and to 3.9\% following the detection of distant metastases (stage IV) (2).

Current methods for diagnosis and monitoring of GC include invasive tests, such as endoscopy and biopsy; however, such tests are limited due to their high cost and invasiveness (3). Esophagogastroduodenoscopy, one of the most reliable methods for diagnosis of GC, is broadly used in China, South Korea and Japan, where the incidence rates of GC are high, while the effectiveness and feasibility of gastroscopy is questionable for countries with low GC incidence rates (2).

In order to screen for GC at an early stage and reduce the incidence and mortality, it is urgent to develop novel biomarkers for early GC diagnosis and prognosis evaluation. Conventional GC-associated serum markers lack of sufficient sensitivity and specificity to obtain early detection, such as carcinoembryonic antigen (CEA), carbohydrate antibody (CA) 12-5, and CA19-9, as well as CA72-4 (4-6), and there is a growing need to identify useful biomarkers for early noninvasive diagnosis and monitoring of the progression of GC. Interleukin-16 (IL-16), produced predominantly by CD8(+) cells, induces chemotaxis of CD4(+) T cells, monocytes and eosinophils. IL-16 acts as an immunomodulatory cytokine that contributes to the regulatory process of CD4(+) cell recruitment and activation at sites of inflammation, and has an essential role in initiating and/or sustaining the inflammatory response (7). In addition, IL-16 can promote the secretion of tumor-associated inflammatory cytokines by monocytes, such as IL-1b, IL- 6 and IL-15, and has an important role in the carcinogenesis of human cancers (8-11). However, the diagnostic and prognostic value of serum IL-16 in patients with GC remains unknown.

In the present study, the mRNA and protein levels of IL-6 in GC cell lines were measured, the IL-6 level in serum from patients with GC was analyzed, and the association of the serum IL-6 level with patient clinical outcomes were evaluated to assess whether serum IL-6 is useful as a diagnostic and prognostic biomarker for GC. 


\section{Materials and methods}

Ethics statement. Informed consent from all patients and healthy controls, and approval from the Affiliated Hospital of Taishan Medical University Ethics Committee was obtained to collect blood samples and review patient medical records.

Sample collection. Patients with GC $(n=98$; mean age, $61.5 \pm 8.1$ years; range $41-75$ years) based on histological examination, received curative treatment in the Affiliated Hospital of Taishan Medical Hospital (Tai'an, China) between January 2005 and March 2010 were enrolled in current study. Prior to the current study, none of the patients with GC had received any chemotherapy, radiation therapy, surgery, immunotherapy or other treatments, and no metastases were identified prior to surgery. Serum samples were collected at the time of diagnosis, prior to tumor resection, at 3 weeks post-operation and at the moment of recurrence. Healthy donors $(n=98)$ were enrolled as control, and they were matched to the patients with GC in terms of sex and age. Serum was obtained from a 5-ml blood sample following centrifugation for $10 \mathrm{~min}$ at $1,006 \mathrm{x} \mathrm{g}$ at room temperature and stored at $-20^{\circ} \mathrm{C}$ prior to analysis.

Follow-up data included clinical, laboratory and radiological examination performed every 3 months during the first year, every 6 months during the subsequent 4 years. Overall survival (OS) was assessed from the date of primary diagnosis to death or last follow-up.

Measurement of serum IL-16 levels. Serum IL-16 levels were determined by the Human IL-16 Quantikine ELISA kit (D1600; R\&D Systems, Inc., Minneapolis, MN, USA) according to the manufacturer's instructions. Briefly, a human IL-16 monoclonal antibody was pre-coated onto a 96-well plate. Standards and samples were added into each well and allowed to incubate for $2 \mathrm{~h}$ at room temperature, and IL-16 was bound by the immobilized antibody. Following washing to remove the unbound substances, an enzyme-linked polyclonal antibody specific for IL-16 was added to each well. Following a wash to remove any unbound antibody-enzyme reagent, a substrate solution was added to the wells and color developed in proportion to the amount of IL-16 bound. The intensity of the colored reaction product was measured using an automated ELISA reader at $450 \mathrm{~nm}$. The results were expressed as ng/ml (7). Serum CEA was measured with ARCHITECT I2000 SR (Abbott Pharmaceutical Co. Ltd., Lake Bluff, IL, USA).

Downregulation of IL-16 mRNA by small interfering RNA (siRNA) transfection. The poorly differentiated human GC cell line MKN45 was obtained from the Cell Bank of the Chinese Academy of Sciences (Shanghai, China). Cells were cultured in RPMI-1640 medium (Thermo Fisher Scientific, Inc., Waltham, MA, USA), supplemented with $10 \%$ fetal calf serum (Gibco; Thermo Fisher Scientific, Inc.) at $37^{\circ} \mathrm{C}$ and $5 \% \mathrm{CO}_{2}$ in humidified incubator. The IL-16 gene silencing by transfection with small interfering RNA (siRNA) was performed according to a previous study (12). GC cells $\left(3 \times 10^{5}\right)$ were washed and resuspended in $100 \mu \mathrm{l}$ OptiMEM I medium
Table I. Sensitivity and specificity of serum IL-16 and CEA.

\begin{tabular}{lccc}
\hline Test & CEA (\%) & IL-16 (\%) & P-value \\
\hline Sensitivity & 68.2 & 79.6 & $<0.05$ \\
Specificity & 59.7 & 78.6 & $<0.05$ \\
\hline
\end{tabular}

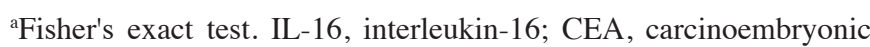
antigen.

(Gibco; Thermo Fisher Scientific, Inc.) For each condition, 50 pmol siRNA (IL-16-targeting siRNAs; HSS142654 and, for the control group, scrambled, non-targeting control siRNA; D-001206-13-20; Invitrogen; Thermo Fisher Scientific, Inc.) was added into the cells followed by incubation at room temperature for $10 \mathrm{~min}$. Lipofectamine 2000 (Thermo Fisher Scientific,Inc.) was diluted 1:40 in OptiMEM I medium without serum and incubated for $10 \mathrm{~min}$ at room temperature, and then the dilution $(100 \mu \mathrm{l})$ was added to the cells and incubated for $20 \mathrm{~min}$ at room temperature. Cell suspensions were placed in a 24-well plate and incubated for $4 \mathrm{~h}$ at $37^{\circ} \mathrm{C}$. Complete medium $(1.5 \mathrm{ml})$ was added into each well and cells were cultured for another $72 \mathrm{~h}$ at $37^{\circ} \mathrm{C}$. Green fluorescence protein was used as a reporter for siRNA. Following transfection, $\geq 5$ random fields of transfected cells were selected by using bright-field microscopy and fluorescent microscopy. Transfection efficiency was determined by dividing the number of fluorescent cells in regions of interest by the total cell number in the field.

RNA extraction and reverse transcription-quantitative polymerase chain reaction $(R T-q P C R)$. Total RNA was extracted from MKN45 cell lines using the TRIzol reagent (Invitrogen; Thermo Fisher Scientific, Inc.) according to the manufacturer's instruction (13). The first-strand IL-16 cDNA was synthesized using the PrimeScript ${ }^{\mathrm{TM}}$ RT-PCR Kit (Takara Bio, Inc., Otsu, Japan) with total RNA. Reverse transcription reactions were carried out at $42^{\circ} \mathrm{C}$ for $60 \mathrm{~min}$ and terminated by heating to $95^{\circ} \mathrm{C}$ for $10 \mathrm{~min}$. RT-qPCR was performed with the SYBR Green PCR MasterMix (Applied Biosystems; Thermo Fisher Scientific, Inc.) under the following conditions: $10 \mathrm{~min}$ at $95^{\circ} \mathrm{C}$, followed by 40 cycles of $15 \mathrm{sec}$ at $95^{\circ} \mathrm{C}$ and $1 \mathrm{~min}$ at $60^{\circ} \mathrm{C}$ ). Each reaction was performed in duplicate. The $2^{-\Delta \Delta \mathrm{Cq}}$ method were used to calculate the relative expression levels, gene expression was normalized to the geometric mean using GAPDH as an internal control (14). The primers used in the qPCR reaction were as follows: IL-16, 5'-GCAAGTCTC TCAAGGGGACC-3' (forward) and 5'-CAGACACACCCC ACACCTTT-3' (reverse); GAPDH, 5'-GACTCATGACCA CAGTCCATGC-3' (forward) and 5'-AGAGGCAGGGATGAT GTTCTG-3' (reverse).

Western blotting. IL-16 protein level was analyzed by western blotting assay according a previous study $(13,15)$. Proteins were extracted in cell lysis buffer (89900; Thermo Fisher Scientific, Inc.) on ice followed by centrifugation at $12,000 \mathrm{x} \mathrm{g}$ for $10 \mathrm{~min}$ at $4^{\circ} \mathrm{C}$, then boiled at $100^{\circ} \mathrm{C}$ for $10 \mathrm{~min}$. Following extraction, the protein concentration was determined by the BCA Protein Assay (Pierce; Thermo Fisher Scientific, Inc.). 

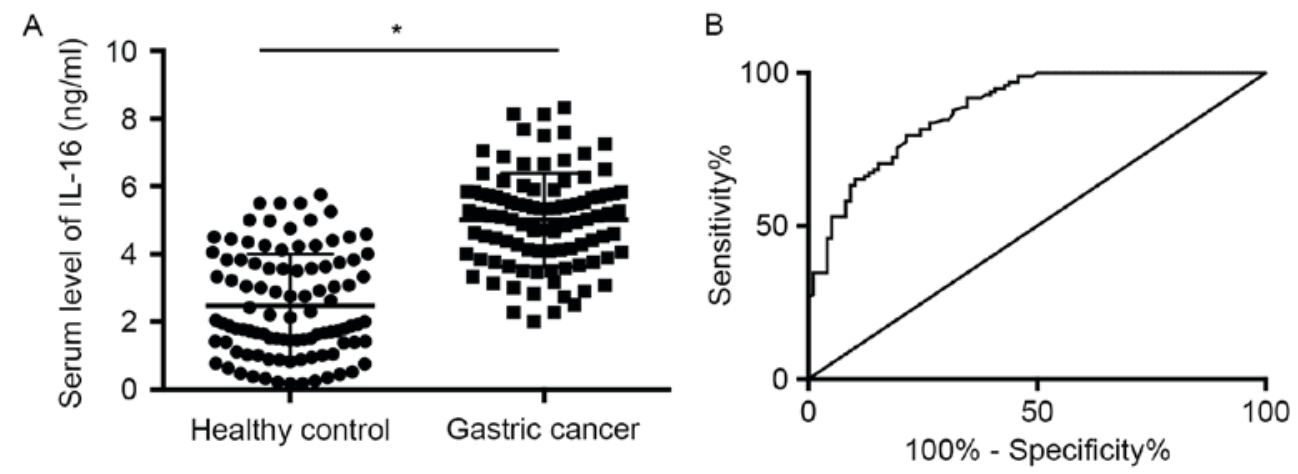

Figure 1. Diagnostic value of serum IL-16 in 98 patients with gastric cancer. (A) Serum IL-16 levels in patients with gastric cancer were higher than that in healthy controls. The statistical significance of the differences was determined using the Mann-Whitney $\mathrm{U}$ test. " $\mathrm{P}<0.05$. (B) The receiver-operating characteristic curves demonstrated the diagnostic strength of IL-16 levels in identifying gastric cancer from healthy controls. IL-16, interleukin-16.

Proteins ( $25 \mu \mathrm{g}$ per well) were resolved by $10 \%$ SDS-PAGE and electro-transferred to nitrocellulose membranes. Each membrane was blocked with $5 \%$ non-fat dried milk in Tris buffered saline-Tween-20 for $1 \mathrm{~h}$ at room temperature, and subsequently incubated with the primary antibody for $16 \mathrm{~h}$ at $4^{\circ} \mathrm{C}$. The following antibodies were used: Anti-IL16 monoclonal (ab180792; 1:1,000; Abcam, Cambridge, UK) and anti- $\beta$ actin (ab8226; 1:1,000; Abcam). Immunoreactivity was detected by sequential incubation with horseradish peroxidase-conjugated secondary antibody (ab97051; 1:1,000; Abcam) for $1 \mathrm{~h}$ at room temperature and visualized using an ECL detection kit (Amersham Pharmacia, Uppsala, Sweden). The density of the specific bands was quantified with an ImageJ version 1.46 (National Institutes of Health, Bethesda, MD, USA).

Cell migration and invasion assays. The siRNA transfection cells and scramble control siRNA transfection cells were used in cell migration and invasion assays (16). The transwell chamber assay was used to assess cell migration ability, and BioCoat ${ }^{\mathrm{TM}}$ Matrigel $^{\mathrm{TM}}$ Invasion Chambers (Corning Incorporated, Corning, NY, USA) were used to analyze the cell invasion ability. Briefly, 200 cells were seeded in the chamber, and 10\% fetal bovine serum (Gibco; Thermo Fisher Scientific, Inc.) was used as the chemoattractant. After $24 \mathrm{~h}$, cells on the lower surface of the chamber were fixed in $10 \%$ paraformaldehyde for $20 \mathrm{~min}$ at room temperature and stained with $0.1 \%$ crystal violet again for $20 \mathrm{~min}$ at room temperature. This was followed by counting under a light microscope. Cells were visualized, and 10 representative fields of view were imaged. Experiments were performed in triplicate at least.

Statistical analysis. Statistical analyses were performed out using SPSS version 17.0 (SPSS, Inc., Chicago, IL, USA) or Prism 6.00 (GraphPad Software, Inc., La Jolla, CA, USA). A receiver-operating characteristic (ROC) curve was obtained and the area under the ROC curve (AUC) was calculated to evaluate the potential diagnosis value of serum IL-16 in GC. The Mann-Whitney U test was used to compare the serum level of IL-16 between patients with GC and healthy volunteers. Wilcoxon signed rank test was used to analyze serum IL-16 levels between pre-operative serum samples and post-operative serum samples. Survival curve data was calculated using the Kaplan-Meier method and compared with log-rank tests. Data

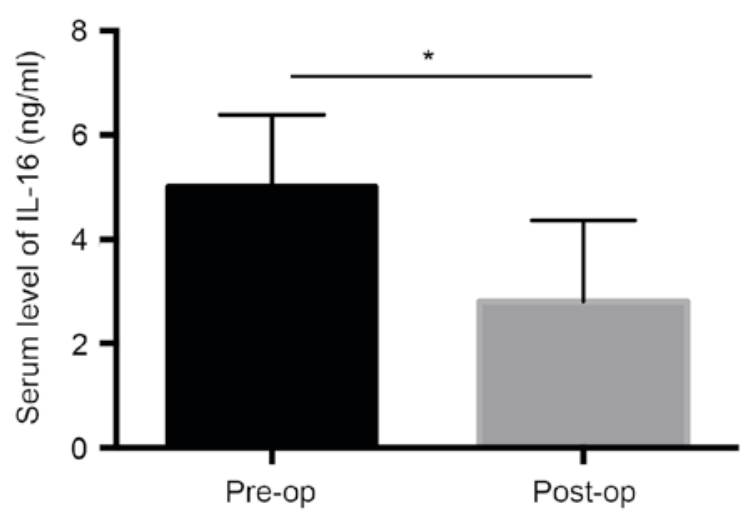

Figure 2. Serum IL-16 levels in patients with gastric cancer pre-op and post-op ( $\mathrm{n}=98$ per group). Serum IL-16 was significantly reduced following surgery. ${ }^{*} \mathrm{P}<0.05$. IL-16, interleukin-16; pre-op, pre-operative; post-op, post-operative.

were presented as mean \pm standard deviation and $\mathrm{P}<0.05$ was considered to indicate a statistically significant difference.

\section{Results}

IL-16 is increased in the serum of 98 patients with GC. The levels of total serum IL-16 in the patients with GC $(n=98)$ and healthy controls $(n=98)$ were detected by ELISA. The serum IL-16 level in the patients with GC were significantly higher compared with those in the healthy controls $(\mathrm{P}<0.05$; Fig. 1A), the mean IL-16 level in the GC group was 2.59-fold higher than that in healthy controls.

In order to analyze the diagnostic potential of serum IL-16 for GC, ROC curves were constructed using on the data from 98 patients with GC and 98 healthy controls. The results indicated the serum IL-16 level was able to differentiate patients with GC $(n=98)$ from healthy controls $(n=98)$ with an AUC of 0.882 .

The sensitivity of IL-16 reached $79.6 \%$, and the specificity was as high as $78.6 \%$, which indicated that for diagnosis of GC IL-16 as a novel marker is better than the traditional tumor marker, CEA, in terms of the sensitivity and specificity (Table I).

Serum IL-16 level is reduced post-operation. In order to evaluate the serum IL-16 level prior to and following operation, 
A

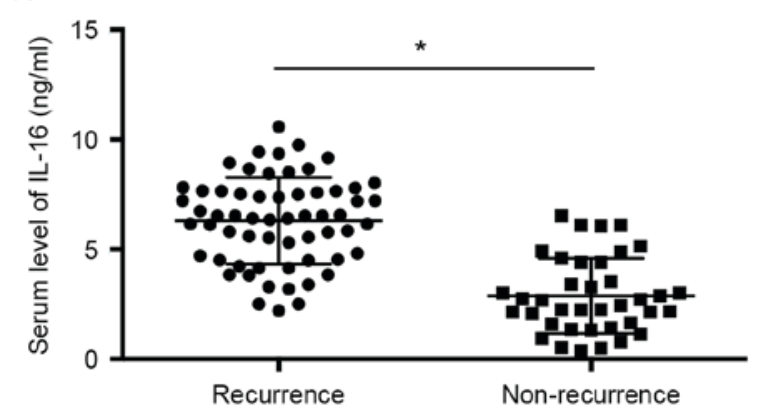

B

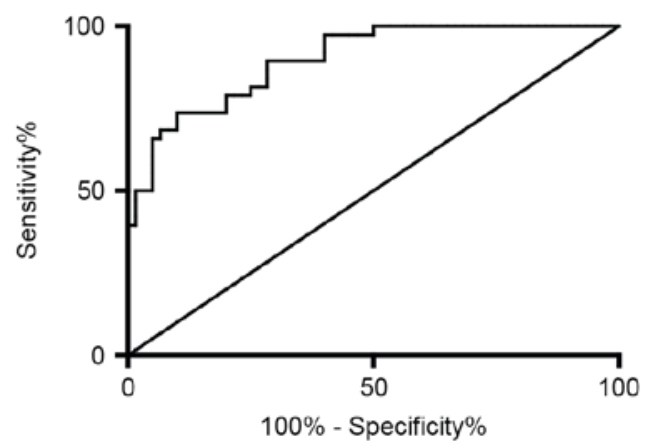

Figure 3. Serum IL-16 levels in patients with and without recurrence. (A) Serum IL-16 levels in the patients with gastric cancer with recurrence were higher compared with no recurrence controls, " $\mathrm{P}<0.05$. (B) Receiver-operating characteristic curve analysis of the serum IL- 16 to discriminate between patients with and without recurrence and non-recurrence. The area under the curve of 0.89 (95\% confidence interval, $0.84-0.95)$ indicates good discriminative power $(\mathrm{P}<0.05)$. IL-16, interleukin-16.

serum samples were obtained pre-operative and at 3 weeks post-operation. As presented in Fig. 2, the serum IL-16 level was significantly reduced following tumor resection, compared with the value prior to tumor resection $(\mathrm{P}<0.05)$, indicating positive association between tumor presence and the serum IL-16 levels in patients with GC.

Serum IL-16 level is increased in patients with recurrence. Fig. 3 demonstrated that serum IL-16 levels in the recurrence group $(n=60)$ were higher than that in the non-recurrence group $(n=38 ; P<0.05)$. ROC curve analysis based on the serum IL-16 level confirmed the ability of IL-16 to distinguish between patients with and without recurrence. The AUC of 0.89 (95\% confidence interval, 0.84-0.95) indicates good discriminative power $(\mathrm{P}<0.001)$.

Low serum IL-16 level predicts good prognosis in patients with $G C$. The correlations between survival and the serum IL-16 levels in patients were evaluated by the Kaplan-Meier method. Patients with GC ( $\mathrm{n}=98)$ were followed-up for 60 months after the tumor resection. Based on the data, the appropriate cut-off value of serum IL-16 levels for predicting the presence of GC was $384 \mathrm{pg} / \mathrm{ml}$. The cut-off value was determined by finding the cut-off with highest Youden Index, or equivalently, the highest Sensitivity + Specificity (17). Kaplan-Meier analysis with the log-rank test indicated that patients with GC in the low serum IL-16 level group $(<384 \mathrm{pg} / \mathrm{ml})$ had a significantly longer 5 -year OS rate compared with the high serum IL-16 level group ( $>384$ pg/ml; P<0.05; Fig. 4).

IL-16 knockdown suppresses the migration and invasion of $G C$ cells. To assess the role of IL-16 in the migration and invasion of GC cells, IL-16 knockdown by siRNA in the GC line MKN45 was performed in the current study. Western blotting assay and RT-qPCR was used to assess IL-16 downregulation in transfected cells, which indicated that siRNA significantly suppressed IL-16 protein levels (Fig. 5A and B).

Transwell assays were performed to evaluate cell migration and invasiveness, and the results revealed that the knockdown of IL-16 suppressed significantly the migration ability and the invasive ability of the GC cells (Fig. 5C and D).

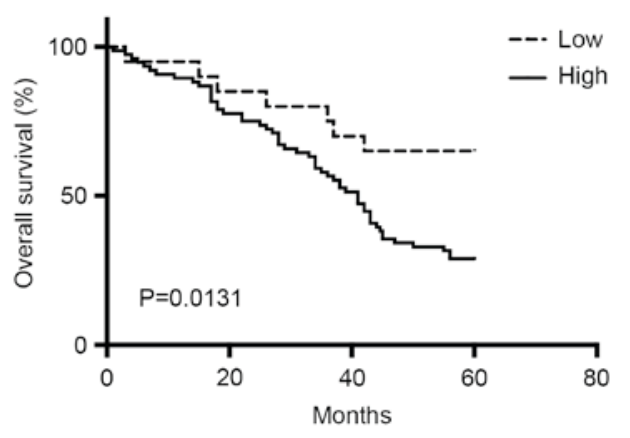

Figure 4. Prognostic value of serum IL-16 in patients with gastric cancer. The 5 -year overall survival rate was $37.8 \%$ for the total study population. Kaplan-Meier analysis revealed that the patients with low serum IL-16 level had longer overall survival than those with high serum IL-16 level $(\mathrm{P}<0.05)$. IL-16, interleukin-16.

\section{Discussion}

GC remains a major public health problem, as it remains the second leading cause of cancer-associated mortality worldwide. An early diagnosis and early treatment strategy can significantly improve the survival rates of patients with GC, and the used of serum biomarkers, including CA 19-9, CEA and CA 72-4, to identify patients with a high risk of GC would improve the early diagnosis rate (18-21). However, the sensitivity and specificity of these serum biomarkers used in diagnosis of GC is low $(22,23)$. Currently, the precise mechanisms underlying GC remain unknown.

Inflammatory disease has been reported as a risk factor for cancer, and $25 \%$ of all cancer cases worldwide correlate with cases of chronic infection and inflammation. Furthermore, patients with chronic inflammation have a much higher risk of tumor formation and progression (24-26). IL-16 is a multifunctional cytokine with a fundamental role in inflammatory diseases, and in the development and progression of tumors (27). Compérat et al (24) reported that IL-16 appears to be a useful prognostic factor in prostate cancer, and its expression in prostate cancer tissue was correlated with tumor aggressiveness and biochemical relapse of the disease. Yellapa et al (25) demonstrated that tissue expression and serum levels of IL-16 increase in association with malignant 
A

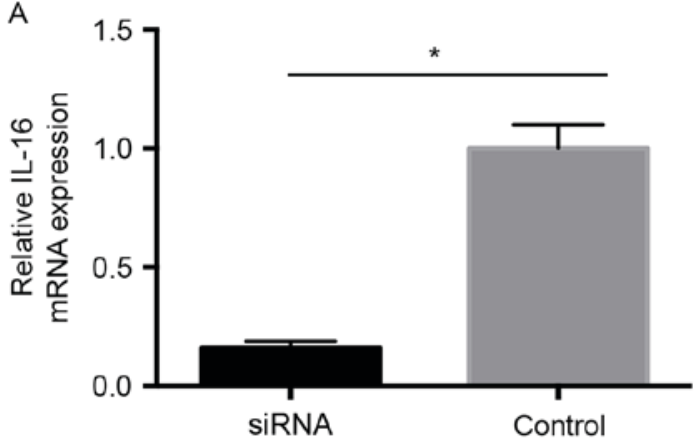

C

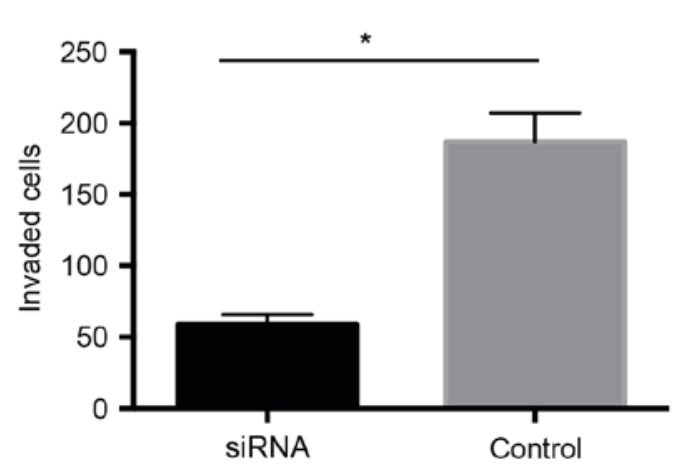

$\mathrm{B}$
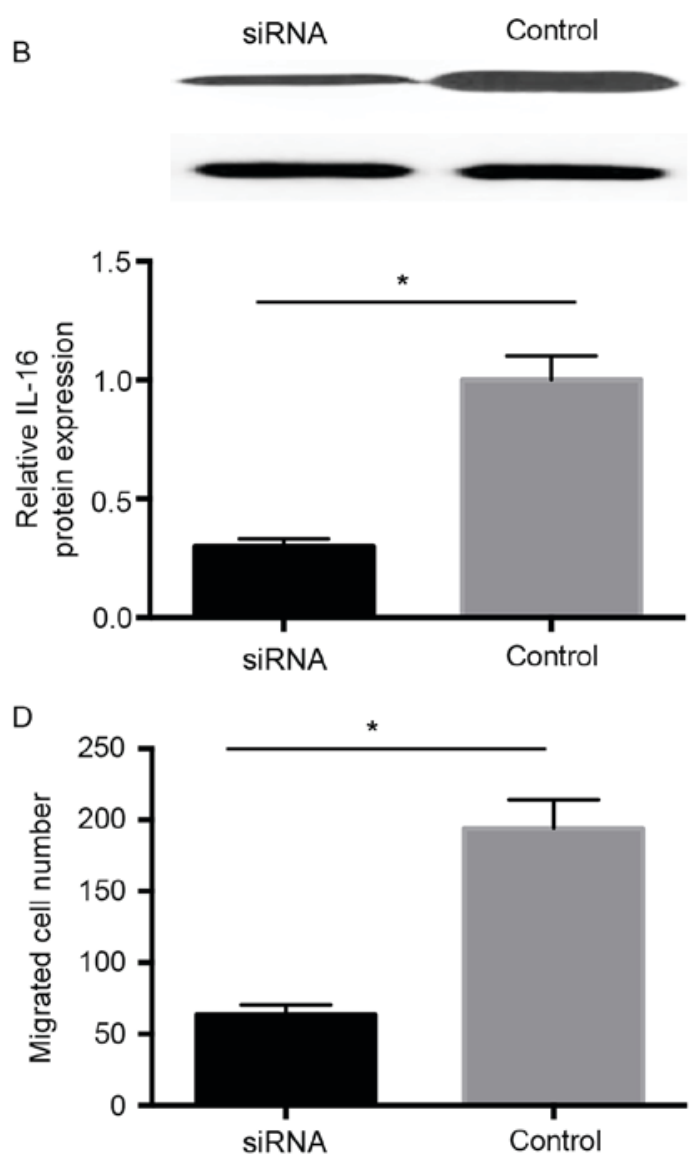

Figure 5. IL-16 knockdown suppressed the migration and invasion of gastric cancer cells. (A) Reverse transcription-quantitative polymerase chain reaction, (B) western blotting, (C) invasion and (D) migration analysis of MKN45 cells transfected with IL-6-targeting siRNA. *P<0.05. IL-16, interleukin-16; siRNA, small interfering RNA.

ovarian tumor development and progression. Regarding GC, a previous study indicated that IL-16 was associated with an increased risk of non-cardia GC in a Chinese population (28).

In the current study, IL-16 expression was decreased in the serum of patients with GC compared with healthy controls, and its expression was associated with diagnostic value and prognosis. IL-16 was useful in the identification of patients with GC from healthy controls, which was superior to the traditional marker, CEA, although the sensitivity of the serum IL-16 as a marker was not particularly high. The IL-16 level in patients with GC was higher than that in healthy controls. Qin et al (27) also reported that the serum IL-16 levels were increased in patients with nasopharyngeal carcinoma compared with controls, which may be because IL-16 is associated with increased susceptibility to nasopharyngeal carcinoma by increasing the production of IL-16. In addition, the serum IL-16 of patients with GC was decreased significantly following tumor resection compared with prior to surgery. As tumor-associated neo-angiogenesis is an early event in tumor development, and IL-16 is a pro-angiogenic cytokine that stimulates production of neoangiogenic factors, decreased serum levels of IL-16 are associated with tumor resection and reduced tumor-associated neo-angiogenesis (29).

Despite improvements in diagnostic procedures and treatment strategies, $>70 \%$ of recurrences and tumor-associated mortality occur within 2 years post-surgery due to early recurrence originating from minimal residual disease. Tumor recurrence is the leading cause of mortality in patients that undergo curative surgery for GC (30). In current study, the IL-16 levels were determined in patients with and without recurrence, and indicated that IL-16 levels were higher in patients with recurrence than in non-recurrence patients, suggesting an association between the serum IL-16 level and tumor recurrence. In addition, ROC curve analysis revealed that serum IL-16 had a diagnostic value in GC, and discriminated between patients with GC recurrence and those with no recurrence.

The 5-year OS rate was $30.8 \%$ in the high IL-16 group, and $65.0 \%$ in the low IL-16 group; Kaplan-Meier analysis revealed that the OS was longer for the patients with low serum IL-16 levels than those with high IL-16 levels.

To further examine the role of IL-16 in the migration and invasion of GC cells, IL-16-depletion in MKN45 cells was established using siRNA, and the cell migration and invasion abilities were assessed using Transwell assays. The knockdown of IL-16 significantly reduced the migration and invasive ability of GC cells.

In conclusion, the current study revealed that serum IL-16 level may not only serve as a useful diagnostic biomarker for patients with GC, but also may predict cancer recurrence and patient prognosis. Furthermore, reducing the IL-16 level may be a novel therapeutic option for treatment of GC. 


\section{References}

1. Hamashima C: Current issues and future perspectives of gastric cancer screening. World J Gastroenterol 20: 13767-13774, 2014.

2. Tong W, Ye F, He L, Cui L, Cui M, Hu Y, Li W, Jiang J, Zhang DY and Suo J: Serum biomarker panels for diagnosis of gastric cancer. Onco Targets Ther 9: 2455-2463, 2016.

3. Liu WL, Liu D, Cheng K, Liu YJ, Xing S, Chi PD, Liu XH, Xue N, Lai YZ, Guo L and Zhang G: Evaluating the diagnostic and prognostic value of circulating cathepsin $\mathrm{S}$ in gastric cancer. Oncotarget 7: 28124-28138, 2016.

4. Huo YR, Huang Y, Liauw W, Zhao J and Morris DL: Prognostic value of carcinoembryonic antigen (CEA), AFP, CA19-9 and CA125 for patients with colorectal cancer with peritoneal carcinomatosis treated by cytoreductive surgery and intraperitoneal chemotherapy. Anticancer Res 36: 1041-1049, 2016.

5. Benini L, Cavallini G, Zordan D, Rizzotti P, Rigo L, Brocco G, Perobelli L, Zanchetta M, Pederzoli P and Scuro LA: A clinical evaluation of monoclonal (CA19-9, CA50, CA12-5) and polyclonal (CEA, TPA) antibody-defined antigens for the diagnosis of pancreatic cancer. Pancreas 3: 61-66, 1988.

6. Kim JH, Jun KH, Jung H, Park IS and Chin HM: Prognostic value of preoperative serum levels of five tumor markers (carcinoembryonic antigen, CA19-9, alpha-fetoprotein, CA72-4, and CA125) in gastric cancer. Hepatogastroenterology 61: 863-869, 2014.

7. Alexandrakis MG,PassamFH,Kyriakou DS, Christophoridou AV, Perisinakis K, Hatzivasili A, Foudoulakis A and Castanas E: Serum level of interleukin-16 in multiple myeloma patients and its relationship to disease activity. Am J Hematol 75: 101-106, 2004.

8. Mathy NL, Scheuer W, Lanzendörfer M, Honold K, Ambrosius D, Norley S and Kurth R: Interleukin-16 stimulates the expression and production of pro-inflammatory cytokines by human monocytes. Immunology 100: 63-69, 2000.

9. Schneider MR, Hoeflich A, Fischer JR, Wolf E, Sordat B and Lahm H: Interleukin-6 stimulates clonogenic growth of primary and metastatic human colon carcinoma cells. Cancer Lett 151: $31-38,2000$.

10. Chung YC and Chang YF: Serum interleukin-6 levels reflect the disease status of colorectal cancer. J Surg Oncol 83: 222-226, 2003.

11. Kai H, Kitadai Y, Kodama M, Cho S, Kuroda T, Ito M, Tanaka S, Ohmoto Y and Chayama K: Involvement of proinflammatory cytokines IL-1beta and IL-6 in progression of human gastric carcinoma. Anticancer Res 25: 709-713, 2005.

12. Atanackovic D, Hildebrandt Y, Templin J, Cao Y, Keller C, Panse J, Meyer S, Reinhard H, Bartels K, Lajmi N, et al: Role of interleukin 16 in multiple myeloma. J Natl Cancer Inst 104: 1005-1020, 2012.

13. Villano V, Di Stadio CS, Federico A, Altieri F, Miselli G, De Palma M, Rippa E and Arcari P: Gastrokine 1 mRNA in human sera is not informative biomarker for gastric cancer. J Negat Results Biomed 15: 14, 2016.

14. Livak KJ and Schmittgen TD: Analysis of relative gene expression data using real-time quantitative PCR and the 2(-Delta Delta C(T)) method. Methods 25: 402-408, 2001.

15. Cicchillitti L, Corrado G, Carosi M, Dabrowska ME, Loria R, Falcioni R, Cutillo G, Piaggio G and Vizza E: Prognostic role of NF-YA splicing isoforms and Lamin A status in low grade endometrial cancer. Oncotarget 8: 7935-7945, 2017.
16. Kramer N, Walzl A, Unger C, Rosner M, Krupitza G, Hengstschläger $\mathrm{M}$ and Dolznig $\mathrm{H}$ : In vitro cell migration and invasion assays. Mutat Res 752: 10-24, 2013.

17. Krzanowski WJ and Hand DJ: ROC curves for continuous data. Chapman \& Hall/CRC monographs on statistics \& applied probability. 2009: Chapman and Hall/CRC.

18. Jia S and Cai J: Update on biomarkers in development of anti-angiogenic drugs in gastric cancer. Anticancer Res 36: 1111-1118, 2016.

19. Matsueda S and Graham DY: Immunotherapy in gastric cancer. World J Gastroenterol 20: 1657-1666, 2014.

20. Guadagni F, Roselli M, Cosimelli M, Ferroni P, Spila A, Cavaliere F, Casaldi V, Wappner G, Abbolito MR, Greiner JW, et al: CA 72-4 serum marker-a new tool in the management of carcinoma patients. Cancer Invest 13: 227-238, 1995.

21. Shimada H, Noie T, Ohashi M, Oba K and Takahashi Y: Clinical significance of serum tumor markers for gastric cancer: A systematic review of literature by the Task Force of the Japanese Gastric Cancer Association. Gastric Cancer 17: 26-33, 2014.

22. Carpelan-Holmström M, Louhimo J, Stenman UH, Alfthan $H$ and Haglund C: CEA, CA 19-9 and CA 72-4 improve the diagnostic accuracy in gastrointestinal cancers. Anticancer Res 22: 2311-2316, 2002

23. Liu X, Qiu H, Liu J, Chen S, Xu D, Li W, Zhan Y, Li Y, Chen Y, Zhou Z and Sun X: Combined preoperative concentrations of CEA, CA 19-9, and 72-4 for predicting outcomes in patients with gastric cancer after curative resection. Oncotarget 7 : 35446-35453, 2016.

24. Comperat E, Roupret M, Drouin SJ, et al: Tissue expression of IL16 in prostate cancer and its association with recurrence after radical prostatectomy. Prostate 70: 1622-1627, 2010

25. Yellapa A, Bitterman P, Sharma S, et al: Interleukin 16 expression changes in association with ovarian malignant transformation. Am J Obstet Gynecol 210: 272 e1-10, 2014.

26. Hussain SP and Harris CC: Inflammation and cancer: An ancient link with novel potentials. Int J Cancer 121: 2373-2380, 2007.

27. Qin X, Peng Q, Lao X, et al: The association of interleukin-16 gene polymorphisms with IL-16 serum levels and risk of nasopharyngeal carcinoma in a Chinese population. Tumour Biol: 35 : 1917-1924, 2014

28. Zhang $\mathrm{T}$ and Wang $\mathrm{H}$ : Variants of interleukin-16 associated with gastric cancer risk. Asian Pac J Cancer Prev 14: 5269-5273, 2013 .

29. Yellapa A, Bahr JM, Bitterman P, Abramowicz JS, Edassery SL, Penumatsa K, Basu S, Rotmensch J and Barua A: Association of interleukin 16 with the development of ovarian tumor and tumor-associated neoangiogenesis in laying hen model of spontaneous ovarian cancer. Int J Gynecol Cancer 22: 199-207, 2012.

30. Kang WM, Meng QB, Yu JC, Ma ZQ and Li ZT: Factors associated with early recurrence after curative surgery for gastric cancer. World J Gastroenterol 21: 5934-5940, 2015. 